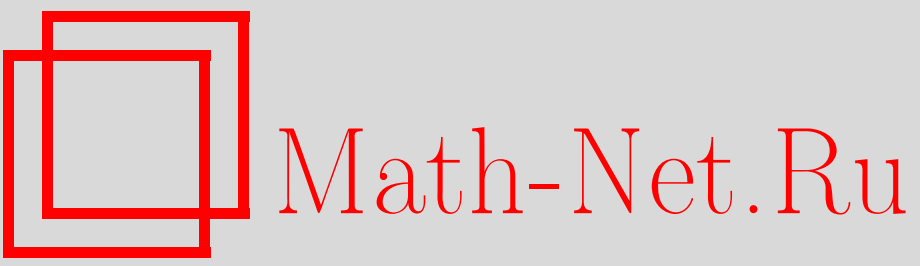

В. Е. Зобов, М. А. Попов, О радиусе сходимости рядов по степеням времени спиновых корреляционных функций гейзенберговского магнетика при высоких температурах, ТМФ, 1997, том 112, номер 3, 479-491

DOI: https://doi.org/10.4213/tmf1057

Использование Общероссийского математического портала Math-Net.Ru подразумевает, что вы прочитали и согласны с пользовательским соглашением

http://www.mathnet.ru/rus/agreement

Параметры загрузки:

IP: 52.205 .19 .152

26 апреля 2023 г., 05:33:46 
ТЕОРЕТИЧЕСКАЯ

И МАТЕМАТИЧЕСКАЯ

ФИЗИКА

Том 112, № 3

сентябрь, 1997

\author{
В.Е. Зобов* ${ }^{*}$ М. А. Попов ${ }^{\dagger}$
}

\title{
О РАДИУСЕ СХОДИМОСТИ РЯДОВ ПО СТЕПЕНЯМ ВРЕМЕНИ СПИНОВЫХ КОРРЕЛЯЦИОННЫХ ФУНКЦИЙ ГЕЙЗЕНБЕРГОВСКОГО МАГНЕТИКА ПРИ ВЫСОКИХ ТЕМПЕРАТУРАХ
}

Исследуется сходимость рядов по степеням времени спиновых автокорреляционных функций гейзенберговского магнетика при бесконечной температуре на решетках разной размерности $d$. По рассчитанным к настоящему времени коэффициентам этих рядов оценены радиусы сходимости. Обнаруженный их рост при уменьшении $d$ объяснен в самосогласованном приближении, для чего предложено и решено отвечающее этому приближению упрощенное нелинейное уравнение для автокорреляционной функции системы с произвольным числом ближайших соседей $Z$. Коэффициенты временного разложения решения представлены в виде деревьев на решетке Бете с координационным числом $Z$. Методом компьютерного моделирования рассчитаны величины этих коэффициентов при размещении деревьев на квадратной, треугольной и простой кубической решетках при условии запрета пересечений ветвей. Эффект исключенного объема, выражающийся в уменьшении этих коэффициентов и в увеличении координаты и показателя степени особенности автокорреляционной функции на оси мнимого времени, усиливается при уменьшении размерности решетки.

\section{1. ВВЕДЕНИЕ}

Вся необходимая информация о наблюдаемых свойствах магнитных систем содержится в спиновых корреляционных функциях, поэтому их расчет является одной из важнейших задач как статистической физики, так и физики магнитных явлений [1-3]. Построение теории таких систем начинают с простых моделей: Изинга, Гейзенберга и т.п. $[2,4]$. К настояшему времени для этих моделей достаточно хорошо разработаны методы расчета статических спиновых корреляционных функций и в меньшей степени динамических. Для изучения собственно динамических свойств спиновых систем и во избежание усложнений, связанных со статическими корреляциями, в теории прибегают к приближению высоких или бесконечных температур [1, 5-13]. Подразумевается при этом, что спины расположены в узлах жесткой решетки. Обоснованием этого приближения служит распространенность спиновых систем (например, ядерных спинов [1]) со

\footnotetext{
* Институт физики им. Л.В. Киренского Сибирского отделения РАН, Красноярск, Россия. E-mail: root@iph.krasnoyarsk.su

${ }^{\dagger}$ Красноярский государственный университет, Красноярск, Россия
} 
слабой по сравнению с реальной температурой тела энергией взаимодействия между магнитными моментами и еще более слабой связью спиновой и решеточной подсистем. Говорить о бесконечной температуре спиновой подсистемы в этом случае допустимо, поскольку у каждого спина имеется конечное число состояний, заселенности которых при такой температуре практически выравнены, и спин в какой-либо момент времени может иметь любое направление. Однако в близкие моменты времени это направление не сохраняется, а изменяется в результате взаимодействий между спинами на временных масштабах, определяемых величиной этого взаимодействия. Потеря со временем памяти о первоначальном направлении спина характеризуется динамическими спиновыми корреляционными функциями, которые сохраняются и при бесконечной температуре спиновой подсистемы. Более того, предсказываемый вид этих функций и их спектров во многих случаях наблюдаем на эксперименте.

В приближении бесконечных температур к настоящему времени известно точное решение для нескольких простых модельных систем: системы с изинговским взаимодействием [1], системы с равным взаимодействием всех спинов [10] и $X Y$-цепочки [8]. В указанных системах происходит или независимая прецессия отдельных спинов в локальных полях (первые два примера), или независимое движение спиновых волн. В общем случае взаимодействие между спинами или спиновыми волнами нарушает независимость их движений, приводя, в частности, к бесконечной системе зацепляющихся уравнений для корреляционных функций или функций Грина $[2,3]$. Основная трудность теории состоит в необходимости выбора какого-либо способа расцепления этой цепочки уравнений для их решения. Сделанньй выбор предопределяет вид получаемых корреляционных функций и их свойства.

В настоящее время благодаря успехам современной теории критических явлений при описании фазовых переходов, свойств полимеров и перколяции, а также развитию вычислительной техники становится реальным иной подход. Он связан с анализом аналитических свойств рассматриваемых динамических спиновых корреляционных функций, в частности с выяснением характеристик их особых точек в плоскости комплексной временной переменной. Знание указанных свойств корреляционных функций позволяет выбрать правильное приближение при решении уравнений для них и в конечном счете повысить точность расчета корреляционных функций и их спектров.

В работах $[12,13]$ было установлено, что автокорреляционная функция гейзенберговского магнетика на решетке бесконечной размерности при бесконечной температуре имеет особые точки на мнимой оси комплексной временной переменной. Еше раньше Араки [14] доказал, что ряд по степеням времени такой корреляционной функции одномерной системы имеет бесконечный радиус сходимости. Нам неизвестны работы, в которых выполнялся бы подобный анализ аналитических свойств временных корреляционных функций двух- и трехмерных спиновых систем. Такому анализу посвящена предлагаемая работа. Для корреляционных функций будут получены оценки радиуса сходимости рядов по степеням времени и характеристик ближайших особых точек. С этой целью во втором разделе используются известные точные значения нескольких первых коэффициентов рядов, в третьем - нелинейные уравнения, полученные в самосогласованном приближении Резибуа-де Ленера-Блюма-Хаббарда [5, 6]. Наконец, в четвертом разделе методом компьютерного моделирования исследовано влияние исключенно- 


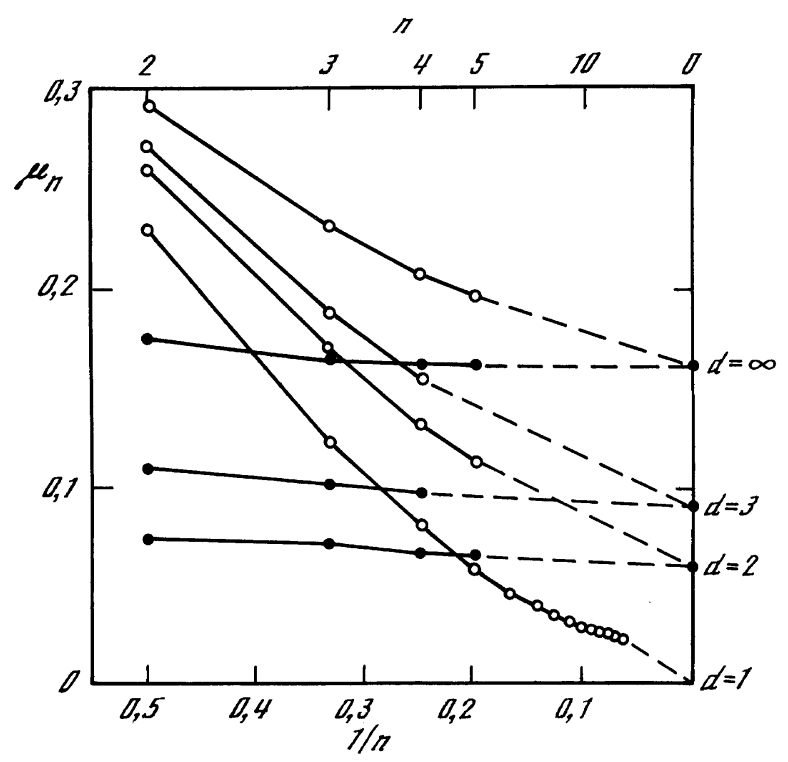

Рис. 1. Отношения коэффициентов ряда по степеням времени для спиновых автокорреляционных функций.

го объема при переходе от спиновых систем на решетках Бете [15, 16], которым соответствует самосогласованное приближение, к двух- и трехмерным решеткам.

\section{2. АНАЛИЗ РЯДОВ ПО СТЕПЕНЯМ ВРЕМЕНИ}

Рассмотрим автокоррелящионную функцию при бесконечной температуре спина, pacположенного в одном из узлов бесконечной решетки размерности $d$,

$$
F(t)=\operatorname{Sp}\left\{\exp (i \mathcal{H} t) S_{0}^{\alpha} \exp (-i \mathcal{H} t) S_{0}^{\alpha}\right\} / \operatorname{Sp}\left\{\left(S_{0}^{\alpha}\right)^{2}\right\},
$$

где $\mathcal{H}$ - гамильтониан изотропной модели Гейзенберга со взаимодействием между ближайшими соседями, $S_{0}^{\alpha}-\alpha$-компонента $(\alpha=x, y, z)$ векторного оператора спина в узле $0, S=1 / 2$. Корреляционная функция (1) может быть разложена в степенной ряд

$$
F(t)=\sum_{n=0}^{\infty}(-1)^{n} M_{2 n} t^{2 n} /(2 n) !
$$

коэффициенты разложения которого являются моментами спектральной плотности этой функции [1]. Второй момент или средний квадрат частоты равен

$$
M_{2}=2 \sum_{j} J_{i j}^{2}=2 J^{2} Z,
$$

где $J_{i j}$ - константа взаимодействия (обменный интеграл) между спинами в узлах решетки $i$ и $j, Z$ - число ближайших соседей, в частности $Z=2 d$ для простой кубической решетки размерности $d$. Второй момент задает временной масштаб всех величин.

5 Теоретическая и математическая физика, т. 112, № 3, 1997 г. 
При необходимости исключения связанной с ним зависимости от $d$ в дальнейшем будем использовать безразмерные величины $t \sqrt{M_{2}}, M_{2 n} / M_{2}^{n}$ и т.д. Расчет моментов высокого порядка сталкивается с большими трудностями: известны моменты до $n=4$ при $d=3$ [7], до $n=5$ при $d=2$ [9] и $d=\infty$ [13], до $n=15$ при $d=1$ [11].

Радиус сходимости $\tau_{Z}$ ряда (2), следуя [4], можно оценить с помощью предела $\mu$ отношения соседних членов ряда

$$
\mu_{n}=M_{2 n} /\left[M_{2 n-2} M_{2} 2 n(2 n-1)\right]
$$

при $n \rightarrow \infty$, поскольку $\mu=1 /\left(\tau_{Z}^{2} M_{2}\right)$. Последовательности отношений $(3)$, рассчитанные по известным моментам, приведены на рис. 1 , где светлые кружки изображают отношения коэффициентов ряда по степеням времени для спиновых автокорреляционных функций в случае простых кубических решеток разной размерности. Те же отношения, пересчитанные с учетом показателей особенностей $\left(p_{\infty}=2, p_{6}=3, p_{4}=4\right)$, изображены темными кружками. При $n=\infty$ показаны предельные значения $\mu$, соответствуюшие (15) (см. ниже). С уменьшением размерности решетки последовательности отношений (3) смешаются вниз, следовательно, оцененный по ним радиус сходимости $\tau_{Z} \sqrt{M_{2}}$ увеличивается. Причем, поскольку масштабная зависимость от $Z$ через $M_{2}$ уже учтена после перехода к безразмерным величинам, то здесь налицо дополнительная зависимость от $d$.

\section{3. САМОСОГЛАСОВАННЫЕ УРАВНЕНИЯ}

В причине появления обнаруженной зависимости попробуем разобраться, взяв в качестве основы теорию кинетических уравнений $[5,6,13]$, приводящую в приближении большого числа соседей $Z$ к нелинейному интегральному уравнению для автокорреляционной функции (1):

$$
\frac{d}{d t} F(t)=-\int_{0}^{t} G\left(t-t^{\prime}\right) F\left(t^{\prime}\right) d t^{\prime},
$$

где $G(t)$ - функция памяти или ядро уравнения, представленное в виде ряда по одетым неприводимым скелетным диаграммам с возрастаюшим числом вершин. Сохранив только первый член этого ряда, получим

$$
\frac{d}{d t} F_{i i}(t)=-2 \sum_{j} J_{i j}^{2} \int_{0}^{t} F_{i i}\left(t^{\prime}\right) F_{j j}\left(t^{\prime}\right) F_{i i}\left(t-t^{\prime}\right) d t^{\prime}
$$

где для удобства дальнейшего анализа в автокорреляционных функциях спинов $i$ и $j$ добавлен в обозначениях соответствующий индекс, который в решетке из одинаковых спинов обычно опускают.

Диаграммный ряд можно перегруппировать иначе в виде кумулянтного разложения, сохранение первого члена которого ведет к уравнению [6]

$$
F_{i i}(t)=\exp \left\{-2 \sum_{j} J_{i j}^{2} \int_{0}^{t}\left(t-t^{\prime}\right) F_{j j}\left(t^{\prime}\right) d t^{\prime}\right\}
$$


Выполненный нами анализ [13] показал, что при $d \rightarrow \infty$ использованная в (4)-(6) процедура одевания является строгой, а уравнение (4) совпадает с уравнением для корреляционной функции магнитного момента, прецессирующего во флуктуирующем гауссовском магнитном поле, корреляционная функция которого выражается через искомую корреляционную функцию. Сложньй вид этого нелинейного интегрального уравнения связан с неперестановочностью поворотов магнитного момента вокруг не совпадающих в разные моменты времени мгновенных ориентаций флуктуирующего магнитного поля. Упрощение в (6) достигнуто за счет пренебрежения этой неперестановочностью поворотов или путем изменения ориентации поля. Физической аргументацией такого приближения, получившего название адиабатического [1], служит медленность изменения ориентации поля.

В рассмотренном в [13] пределе $d \rightarrow \infty$ решения уравнений (5) и (6) на оси мнимого времени являются соответственно минорантой и мажорантой решения уравнения (4), поскольку в (5) неприводимые скелетные диаграммы взяты с заниженными коэффициентами по сравнению с (4), а в (6) - с завышенными. Заметим, что если в (6) коэффициент 2 заменить на 1, то те же диаграммы войдут уже с заниженными коэффициентами. Для нас сейчас важно, что решения всех этих уравнений имеют особые точки на оси мнимого времени. Различия между ними носят количественный характер и заключаются в разных значениях координат особых точек и коэффициентах в главных частях функций.

Формально в уравнениях (4)-(6) содержится зависимость от $Z$ через масштабный множитель $M_{2}$. Однако сами эти уравнения в таком виде получены в пределе $d \rightarrow \infty$. Попробуем теперь качественно учесть изменения уравнений при переходе к конечным $d$. По самой логике одевания неприводимых скелетных диаграмм не следует одновременно использовать взаимодействие с одинаковыми спинами при одевании разных линий, поскольку это ведет к диаграмме с большим числом вершин. В [5] от этого ограничения отказываются, подразумевая, что соответствуюшая корректировка величиной $1 / Z$ может быть сделана в коэффищиентах диаграмм с большим числом вершин. Анализ подобных поправок, получивших название объемных взаимодействий, в теориях перколяции и полимеров $[16,17]$ показал, что в двух- и трехмерных системах эти поправки ведут к расходимостям. Поэтому мы поступим иначе и будем запрешать такие вклады сразу же при их появлении.

Обратимся к уравнению (5) и рассмотрим слагаемое со взаимодействием спинов $i$ и $j$. При одевании линии $i$ следует отбрасывать взаимодействие со спином $j$, а при одевании линии $j$ - со спином $i$. Кроме того, надо исключать взаимодействие спинов $i$ и $j$ с общим спином $k$. Временно предположим, что у спинов $i$ и $j$ нет общих соседей. Это имело бы место при рассмотрении спиновых систем на решетке Бете [15, 18], с которых мы и начнем. Переход к реальным решеткам будет сделан позже.

Вернемся к гейзенберговской модели с равным взаимодействием $Z$ соседей. Тогда с учетом сделанных ограничений вместо уравнения (5) имеем

$$
\frac{d}{d t} F_{k}(t)=-2 J^{2} Z \int_{0}^{t}\left[F_{Z-1}\left(t^{\prime}\right)\right]^{2} F_{k}\left(t-t^{\prime}\right) d t^{\prime},
$$

где $F_{k}(t)$ - коррелящионная функция спина, у которого оставлено $k$ взаимодействий из $Z$. Последовательность этих функций с уменьшаюшимися от $Z$ до 1 значениями $k$ образует 
систему из $Z$ уравнений

$$
\frac{d}{d t} F_{k}(t)=-2 J^{2} k \int_{0}^{t} F_{Z-1}\left(t^{\prime}\right) F_{k-1}\left(t^{\prime}\right) F_{k}\left(t-t^{\prime}\right) d t^{\prime}
$$

главная часть решения которой в окрестности особой точки на оси мнимого времени определена в приложении методом Пенлеве в сочетании с методом моментов [13, 18-20].

Перейдем теперь к более простому, допускаюшему аналитическое решение уравнению (6), записав его с учетом сделанных предположений в виде

$$
F_{Z}(t)=\left[F_{1}(t)\right]^{Z}
$$

где $F_{1}(t)$ - вклад в корреляционную функцию от взаимодействия с одним спином окружения, для которого в рамках данного подхода выполняется уравнение

$$
F_{1}(t)=1-2 J^{2} \int_{0}^{t} d t_{1} \int_{0}^{t_{1}} d t_{2}\left[F_{1}\left(t_{2}\right)\right]^{Z-1}
$$

Уравнение (10) эквивалентно дифференциальному уравнению

$$
\ddot{F}_{1}=-2 J^{2} F_{1}^{Z-1}
$$

При $Z \rightarrow \infty$ уравнение (9) с учетом (10) переходит в уравнение $(6)$.

Искомый радиус сходимости ряда (2) определяется ближайшей особенностью на оси мнимого времени, поэтому в уравнениях (10) и (11) сделаем замену переменных $t=i \tau$. Решение уравнения (11) представимо в виде

$$
\tau=\frac{\sqrt{Z}}{2 J} \int_{0}^{F_{1}}\left(x^{Z}-1\right)^{-1 / 2} d x
$$

В особой точке $\tau=\tau_{Z}$, а $F_{1}=\infty$. Из (12) следует, что

$$
\tau_{Z}=\tau_{\infty} \frac{\Gamma(1 / 2-1 / Z)}{\sqrt{\pi} \Gamma(1-1 / Z)}
$$

где $\Gamma(x)$ - гамма-функция, $\tau_{\infty}=\pi\left(2 M_{2}\right)^{-1 / 2}-$ координата ближайшей особенности в пределе $Z \rightarrow \infty$, найденная в работе [6]. На основании (12) и (13) можно получить в окрестности особенности для главной части решения следуюшее представление:

$$
F_{1}(t)=c\left(i t+\tau_{Z}\right)^{-p}
$$

где

$$
p=2 /(Z-2), \quad c=\left\{\sqrt{2} Z /\left[\sqrt{M_{2}}(Z-2)\right]\right\}^{p} .
$$

При уменьшении $Z$ координата особенности, измеренная в единицах $\sqrt{M_{2}}$, увеличивается и принимает бесконечное значение при $Z=2$ в одномерных системах. Такая 
зависимость качественно согласуется с выводами, сделанными выше по последовательности отношений (3), и с теоремой Араки [14]. Можно проверить, что указанные качественные свойства присуши решению системы уравнений (8), найденному в приложении. Поэтому в дальнейших сложных расчетах мы ограничимся простейшими уравнениями (9)-(12). Для улучшения количественных результатов в формуле $(8)$ вместо $\tau_{\infty}$ возьмем значение, найденное в [13] для решения полного уравнения, $\tau_{0}=2.49 / \sqrt{M_{2}}$. В результате получаем интерполяционную формулу, которая, в частности, дает

$$
\tau_{4}=4.16 / \sqrt{M_{2}}, \quad \tau_{6}=3.33 / \sqrt{M_{2}} .
$$

Соответствуюшие этим значениям точки для $\mu$ показаны на рис. 1.

С учетом ожидаемого показателя степени особенности (14) можно улучшить сходимость последовательности отношений в формуле $(3)[4,13]$, заменив $2 n(2 n-1)$ на $\left(2 n+p_{Z}-1\right)\left(2 n+p_{Z}-2\right)$, где $p_{Z}=Z p$. На рис. 1 показаны такие отношения при $p_{4}=4$ для $d=2$ и $p_{6}=3$ для $d=3$. Видно, что результаты формулы $(8)$ с $\tau_{0}$ неплохо согласуются с последовательностями значений отношений.

\section{4. ВЛИЯНИЕ ИСКЛЮЧЕННОГО ОБЪЕМА}

В уравнениях (9) и (10), как и в уравнениях (6) Блюма-Хаббарда [6], сохранена независимость движения спинов, создаюших поле. Для такой независимости нужно, чтобы у разных спинов были свои соседи или, другими словами, чтобы действие каждого спина на выделенный передавалось своим собственным отдельным путем. Этому условию, как уже отмечалось выше, удовлетворяют решетки Бете [15]. Тем самым уравнения (9) - (11) соответствуют спиновой системе на решетке Бете с координационным числом $Z$ [18]. В системах на реальных двух- и трехмерных решетках действие далекого спина на выделенный может передаваться уже несколькими путями. Применяя уравнения (9)-(11) к таким системам, мы все эти различные пути приписываем как бы не одному, а нескольким спинам, поэтому завышаем вклады от далеких спинов. Для оценки возможного влияния этого завышения на изучаемые особенности корреляционных функций рассмотрим моменты в обоих случаях.

Моменты корреляционных функций (9)-(11) проще всего найти методом рекуррентных уравнений $[13,19,20]$. Первые 11 моментов из рассчитанных таким способом по уравнению (П.6) (см. приложение) даны в таблице. В этой таблице приведены моменты $M_{2 n}$ автокорреляционных функций в приближении деревьев для квадратной (sq), треугольной $(\operatorname{tr})$ и простой кубической $(\mathrm{sc})$ решеток, а также решеток Бете с теми же координационными числами $Z$ (в единицах $\left.\left(2 J^{2}\right)^{n}\right)$.

В обшем случае (1) момент порядка $2 n$ определяется через шпур от $2 n$-кратного коммутатора гамильтониана со спиновым оператором $S_{0}^{\alpha}$. Если каждой паре взаимодействуюших спинов в гамильтониане ставить в соответствие связь между соответствующими узлами на решетке, то результат может быть представлен в виде суммы по решеточным фигурам из связей $f[7]$

$$
M_{2 n}=\left(2 J^{2}\right)^{n} \sum_{f} N(f) K_{2 n}(f)
$$




\begin{tabular}{|c|c|c|c|c|c|}
\hline \multirow{2}{*}{} & \multicolumn{3}{|c|}{$Z=4$} & \multicolumn{3}{c|}{$Z=6$} \\
\cline { 2 - 6 } & $\mathrm{sq}$ & Бете & $\mathrm{tr}$ & $\mathrm{sc}$ & Бете \\
\hline 1 & 4 & 4 & 6 & 6 & 6 \\
2 & 48 & 48 & 120 & 120 & 120 \\
3 & 1008 & 1008 & 4368 & 4560 & 4560 \\
4 & 31744 & 32256 & 240000 & 276864 & 278400 \\
5 & 1370944 & 1463616 & $1.810 \times 10^{7}$ & $2.437 \times 10^{7}$ & $2.492 \times 10^{7}$ \\
6 & $7.642 \times 10^{7}$ & $8.943 \times 10^{7}$ & $1.769 \times 10^{9}$ & $2.915 \times 10^{9}$ & $3.076 \times 10^{9}$ \\
7 & $5.280 \times 10^{9}$ & $7.075 \times 10^{9}$ & $2.154 \times 10^{11}$ & $4.525 \times 10^{11}$ & $5.007 \times 10^{11}$ \\
8 & $4.392 \times 10^{11}$ & $7.038 \times 10^{11}$ & $3.175 \times 10^{13}$ & $8.809 \times 10^{13}$ & $1.039 \times 10^{14}$ \\
9 & $4.307 \times 10^{13}$ & $8.598 \times 10^{13}$ & $5.547 \times 10^{15}$ & $2.096 \times 10^{16}$ & $2.677 \times 10^{16}$ \\
10 & $4.898 \times 10^{15}$ & $1.266 \times 10^{16}$ & - & - & $8.389 \times 10^{18}$ \\
11 & $6.377 \times 10^{17}$ & $2.210 \times 10^{18}$ & - & - & $3.140 \times 10^{21}$ \\
\hline
\end{tabular}

где $N(f)$ - число размешений данной фигуры на решетке, $K_{2 n}$ - вклад в момент, соответствуюший данной фигуре (спиновый сомножитель). Для корреляционных функций, описываемых уравнениями (9) и (10), возможными фигурами являются корневые деревья из $n$ связей на решетке Бете с координационным числом $Z$. Спиновый сомножитель для каждого такого дерева есть произведение сомножителей его узлов. Сомножитель узла, из которого выходит $k$ ветвей, содержаших соответственно $n_{1}, n_{2}, \ldots, n_{k}$ связей, равен

$$
\frac{\left(2 n_{1}+2 n_{2}+\cdots+2 n_{k}\right) !}{\left(2 n_{1}\right) !\left(2 n_{2}\right) ! \ldots\left(2 n_{k}\right) !} .
$$

Перенесем теперь деревья, построенные на решетке Бете, на простую кубическую, квадратную и треугольную решетки. Треугольная решетка интересна тем, что у нее размерность, как у квадратной решетки, а число ближайших соседей, как у простой кубической решетки. Поэтому треугольная решетка позволяет выяснить, какая из этих характеристик определяет интересуюшие нас параметры. Часть деревьев удается разместить только при повторном использовании одних и тех же узлов и связей, т.е. при допушении самопересечений. Другая часть деревьев может быть размешена без самопересечений. $\mathrm{K}$ указанному вьше переучету вкладов спинов приводят деревья с самопересечениями, поэтому мы сохраним в (16) только вклад от деревьев без самопересечений. Отметим, что изменение свойств решеточных фигур общего вида при наложении условия запрета пересечений получило название эффекта исключенного объема. Этот эффект на деревьях ранее изучался, например, в работах по теории полимеров [21, 22]. Сейчас мы его хотим изучить в динамической теории спиновых систем. Основной метод решения таких задач - компьютерное моделирование. 


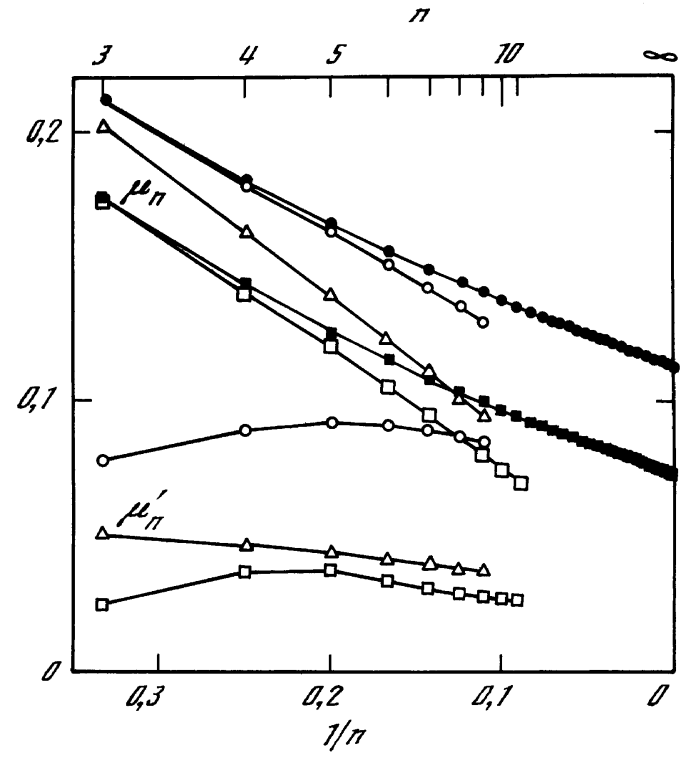

Рис. 2. Отношения коэффициентов ряда по степеням времени для спиновых автокорреляционных функций в приближении деревьев $\mu_{n}$ и линейные экстраполяции этих отношений $\mu_{n}^{\prime}$.

Расчет проводился по следуюшей схеме. Строилось дерево из $n$ связей без самопересечений и вычислялся для него спиновый сомножитель, совпадающий с сомножителем соответствуюшего дерева на решетке Бете. Перебирались все различные такие деревья с $n$ связями. Сумма их спиновых сомножителей дает величину искомого момента. Рассчитанные моменты приведены в таблице, а найденные по ним последовательности отношений (3) показаны на рис. 2 . Во всех случаях зависимость $\mu_{n}$ от $1 / n$ близка к линейной. Предельное значение $\mu$ можно оценить при помоши линейной экстраполящии [4]

$$
\mu_{n}^{\prime}=n \mu_{n}-(n-1) \mu_{n-1}
$$

Последовательности этих значений показаны также на рис. 2. Из рисунка видно, что уменьшение отношений вследствие учета эффекта исключенного объема значительнее у двумерных решеток. Причем после учета этого эффекта результаты для треугольной решетки (светлые треугольники) при больших $n$ отдалились от таковых для кубической (светлые кружки) и приблизились к результатам для квадратной решетки (светлые квадраты). $\mu_{n}$ для решеток Бете с $Z=2 d$ изображены на рис. 2 темными кружками и квадратами.

Определим $\mu$ по последней точке последовательностей (17):

$$
\mu=\frac{1}{\tau_{Z}^{2} M_{2}} \approx \begin{cases}0.027 & \text { для квадратной решетки, } \\ 0.037 & \text { для треугольной решетки, } \\ 0.085 & \text { для простой кубической решетки. }\end{cases}
$$


Из сравнения (18) с (13) следует, что при учете исключенного объема радиус сходимости $\tau_{Z} \sqrt{M_{2}}$ увеличился примерно в 1.7 раза при $d=2(1.65$ для квадратной и 1.74 для треугольной решеток) и в 1.2 раза при $d=3$. Соответствуюшее увеличение следует ожидать и для значений радиуса (15), оцененных на рис. 1 по начальным моментам, для которых исключенный объем еще не проявил себя. Отметим, что в точных моментах эффект исключенного объема может оказаться слабее, поскольку там допускается многократное взаимодействие с одним и тем же спином (конечно, с другим коэффициентом, чем это получалось выше при пересечении ветвей дерева). Мы исключили такое взаимодействие с тем, чтобы выяснить принципиальный вопрос о сходимости ряда. Дело в том, что сингулярность возможна только у бесконечных систем, как это хорошо известно из современной теории критических явлений, т.е. при участии бесконечного числа спинов. Повторное участие одного и того же спина в этом смысле бесполезно, хотя изменяет координату особенности, если таковая возникла.

Другим проявлением исключенного объема является увеличение показателя степени $p_{Z}$, как это видно на рис. 2 по возрастанию крутизны спада последовательности отношений. Возврашаясь к результатам (12)-(14), легко проверить, что оба эти проявления исключенного объема качественно воспроизводятся заменой $Z$ на меньшее число $Z^{\prime}$. Это можно интерпретировать как результат взаимодействия ветвей дерева из-за условия запрета пересечений, выражаюшегося в уменьшении реального числа ответвлений в узлах по сравнению с их значением у деревьев без взаимодействия. Будем обозначать указанные величины через $\left\{p_{\mathrm{sq}}, Z_{\mathrm{sq}}\right\},\left\{p_{\mathrm{tr}}, Z_{\mathrm{tr}}\right\}$ и $\left\{p_{\mathrm{sc}}, Z_{\mathrm{sc}}\right\}$ соответственно для квадратной, треугольной и простой кубической решеток. На основании последовательности отношений $\mu_{n}$ мы оцениваем величину $p_{\mathrm{sc}}$ в интервале от 3.5 до $5, p_{\mathrm{tr}}-$ в интервале от 12 до 16, a $p_{\mathrm{sq}}$ - в интервале от 10 до 15. Для повышения точности нужно знать больше моментов, но уже из этих грубых оценок видно различие величин показателей степеней особенностей для двух- и трехмерных решеток. Далее, из определения $p_{Z}=2 Z /(Z-2)$ получаем оценки соответствуюших значений $Z^{\prime}: Z_{\mathrm{sc}}=3.3 \div 4.7, Z_{\mathrm{tr}}=2.3 \div 2.4$, $Z_{\mathrm{sq}}=2.3 \div 2.5$. При таких $Z^{\prime}$ уравнение $(10)$ остается нелинейным, хотя степень нелинейности $(Z-1)$ уменьшается. Природа нелинейности кроется в том, что и на реальной решетке при больших $n$ дерево может быть разбито на участки, рост которых происходит независимо друг от друга. На решетке Бете такими участками являются все $(Z-1)$ ветвей, выходящих из одного узла.

\section{5. ЗАКЛЮЧЕНИЕ}

Анализ доступных первых членов ряда по степеням времени спиновой автокорреляционной функции гейзенберговского магнетика при бесконечной температуре дает основания полагать, что радиус сходимости ряда имеет конечное значение для двух- и трехмерных решеток. Его величина, выраженная в единицах $\sqrt{M_{2}}$, растет при уменьшении размерности. Здесь играют роль два обстоятельства, ведущие к уменьшению степени нелинейности уравнения для корреляционной функции: уменњшение координационного числа решетки и усиление эффекта исключенного объема.

Точные уравнения, описывающие изменение автокорреляционной функции во времени, сложнее приближенных уравнений (4)-(10). Однако свойство нелинейности уравнений, приведшее к появлению особенности их решений на оси мнимого времени, по на- 
шему мнению, должно сохраниться. Оно отражает независимость движения магнитных моментов разнесенных в пространстве частей системы, следуюшую из того, что при $T=\infty$ статические корреляции отсутствуют, а динамические коррелящии являются короткодействующими. В подтверждение этого свойства можно сослаться также на экспериментальные данные. Дело в том, что одним из следствий наличия указанных особенностей корреляционных функций на оси мнимого времени являются экспоненциальные высокочастотные асимптотические разложения их спектров. Экспериментально наблюдаемые крылья спектров оказываются близкими по форме к экспоненциальным (см. их анализ в $[13,19,20])$. Поскольку основная часть экспериментов выполнена методом ядерного магнитного резонанса (ЯМР) на системах с диполь-дипольным взаимодействием, в настояшее время нами ведется работа над обобщением развитой выше теории на анизотропный случай.

Работа выполнена при финансовой поддержке Красноярского краевого фонда науки, грант 5F0068.

\section{ПРИЛОЖЕНИЕ}

Определим главную часть решения системы уравнений (8) в окрестности особой точки с координатой $\tau_{Z}$, используя метод, аналогичный методу Пенлеве в теории нелинейных обыкновенных дифференциальных уравнений. С этой целью возьмем эту главную часть в виде

$$
F_{k}(t) \approx c_{k}\left(i t+\tau_{Z}\right)^{-m_{k}}
$$

подставим в (8) и сохраним в правой части только основной член. Из условия равенства левой и правой частей полученных уравнений:

$$
c_{k} m_{k}\left(i t+\tau_{Z}\right)^{-m_{k}-1}=2 J^{2} k c_{Z-1} c_{k-1}\left(m_{Z-1}+m_{k-1}-1\right)^{-1}\left(i t+\tau_{Z}\right)^{1-m_{Z-1}-m_{k-1}} \text {, }
$$

следует система алгебраических уравнений для $c_{k}$ и $m_{k}$, решив которую, находим

$$
\begin{aligned}
m_{k} & =k m_{1}, \quad m_{1}=2 /(Z-2), \\
c_{k} & =\left(c_{Z-1} / m_{1}\right)^{k} / \prod_{n=1}^{k}\left(m_{1} n+1\right), \\
c_{Z-1} & =\left\{\left[m_{1} /(Z-2)\right]^{Z-1} \prod_{n=1}^{Z-1}(3 Z-2 n-2)\right\}^{1 /(Z-2)} .
\end{aligned}
$$

В частном случае $k=Z-2$ имеем

$$
c_{Z-2}=m_{1}\left[(Z-1) m_{1}+1\right] \text {. }
$$

Координаты двух ближайших особых точек в силу четности автокорреляционных функций, расположенных на оси мнимого времени симметрично относительно начала 
координат, определим методом моментов. Для этого подставим ряды по степеням времени для функций $F_{k}(t)$ в уравнения системы (8) и приравняем коэффициенты при одинаковых степенях времени. Получаем таким путем систему рекуррентных уравнений для моментов

$$
M_{2 n+2}^{(k)}=2 J^{2} k \sum_{q=0}^{n} M_{2 n-2 q}^{(k)} \sum_{m=0}^{q}\left(\begin{array}{c}
2 q \\
2 m
\end{array}\right) M_{2 q-2 m}^{(k-1)} M_{2 m}^{(Z-1)}
$$

решая которую последовательно, найдем моменты нужного порядка. Координата особой точки, равная радиусу сходимости ряда по степеням времени, может быть определена как предел при $n \rightarrow \infty$ последовательностей

$$
\tau_{Z(2 n)}^{(k)}=\left[\frac{M_{2 n-2}^{(k)} \Gamma\left(2 n+m_{k}\right)}{M_{2 n}^{(k)} \Gamma\left(2 n-2+m_{k}\right)}\right]^{1 / 2}
$$

или

$$
\tau_{Z(2 n)}^{(k)}=\left[\frac{2 c_{k} \Gamma\left(2 n+m_{k}\right)}{M_{2 n} \Gamma\left(m_{k}\right)}\right]^{1 /\left(2 n+m_{k}\right)}
$$

Формулы (П.4) и (П.5) являются уточненными (с учетом известной степени сингулярности $\left.m_{k}[4]\right)$ формулами Д'Аламбера и Коши, соответственно.

В частности, для простейшей системы из двух нелинейных уравнений при $Z=3$ находим указанным путем $\tau_{3} \approx 5.35 / M_{2}^{1 / 2}$. При $Z=2$ получаются линейные уравнения и радиус сходимости рядов становится бесконечным.

В случае уравнений (9)-(11) также могут быть получены рекуррентные уравнения для моментов:

$$
\begin{aligned}
M_{2 n+2}^{(1)} & =2 J^{2} M_{2 n}^{(Z-1)}, \\
M_{2 n}^{(k)} & =\sum_{q=0}^{n}\left(\begin{array}{c}
2 n \\
2 q
\end{array}\right) M_{2 q}^{(1)} M_{2 n-2 q}^{(k-1)}
\end{aligned}
$$

\section{Список литературы}

[1] А. Абрагам. Ядерный магнетизм. М.: ИИЛ, 1963.

[2] С. В. Тябликов. Методы квантовой теории магнетизма. М.: Наука, 1975.

[3] Д. Н. Зубарев, Ю. Г. Рудой. УФН. 1993. Т. 163. С. 103.

[4] М. Фишер. Природа критического состояния. М.: Мир, 1968.

[5] P. Resibois, M. De Leener. Phys. Rev. 1966. V. 152. P. 305.

[6] M. Blume, J. Hubbard. Phys. Rev. B. 1970. V. 1. P. 3815.

[7] T. Morita. J. Math. Phys. 1971. V. 12. P. 2062.

[8] J. H. H. Perk, H. W. Capel. Physica. A. 1980. V. 100. P. 1.

[9] J. Stolz, U. Brandt. Z. Phys. B. 1989. B. 77. S. 111.

[10] J. M. Liu, G. Muller. Phys. Rev. A. 1990. V. 42. P. 5854.

[11] M. Böhm, H. Leschke. J. Phys. A. 1992. V. 25. P. 1043. 
[12] V. E. Zobov. Phys. Lett. A. 1986. V. 119. P. 315.

[13] B. Е. Зобов. ТМФ. 1988. Т. 77. C. 426.

[14] H. Araki. Commun. Math. Phys. 1969. V. 14. P. 120.

[15] M. E. Fisher, J. W. Essam. J. Math. Phys. 1961. V. 2. P. 609.

[16] J. W. Essam. Rep. Progr. Phys. 1980. V. 43. P. 833.

[17] T. Hara, G. Slade. J. Stat. Phys. 1990. V. 59. P. 1469.

[18] В. E. Зобов. Высокочастотные асимптотики автокорреляционной функции гейзенберговского парамагнетика на решетке Бете. В кн.: ЯМР и динамика спиновых систем. Ред. Э. П. Зеер. Красноярск: Институт физики СО РАН, 1988. С. 3.

[19] A. A. Lundin, A. V. Makarenko, V. E. Zobov. J. Phys. Condens. Matter. 1990. V. 2. P. 10131

[20] В.Е. Зобов, А.А. Лундин. ЖЭТФ. 1994. Т. 106. С. 1097.

[21] J.A.M.S. Duarte, H. J. Ruskin. J. Phys. (Paris). 1981. V. 42. P. 1585.

[22] W. A. Seitz, D. J. Klein. J. Chem. Phys. 1981. V. 75. P. 5190.

Поступила в редакцию 27.II.1997 г.

\section{V.E. Zobov, M.A. Popov \\ ON CONVERGENCE RADIUS OF TIME-POWER SERIES FOR SPIN CORRELATION FUNCTIONS OF THE HEISENBERG MAGNET AT HIGH TEMPERATURE}

The time power-series expansions of the spin autocorrelation functions are investigated for the Heisenberg model on lattices of different dimensions $d$ at infinite temperature. The convergence radii of given series are estimated from known expansion coefficients. It is found that the radius increases with decreasing $d$. This dependence is explained in the self-consistent approach based on a simplified nonlinear equation for the autocorrelation function of a system with arbitrary number of nearest neghbors $Z$ that is derived and solved. The time-expansion coefficients of the solution are represented by bond trees on the Bethe lattice of coordination number $Z$. By using the computer simulation method these coefficients are calculated for self-avoiding trees embedding in square, triangular and simple cubic lattices. Excluded volume effects apprearing in the decrease of these coefficients and in the increase of the coordinate and the exponent of the autocorrelation function singularity on the imaginary time axis are found to intensify with a lattice dimension decreasing. 